\title{
Neutron/Gamma Pulse discrimination analysis of GS10 Lithium glass and EJ-204 plastic scintillators
}

\author{
Hajir Al Hamrashdi ${ }^{1,2}$, Stephen D. Monk ${ }^{1}$ and David Cheneler ${ }^{1}$ \\ ${ }^{1}$ Engineering Department, Lancaster University \\ Lancaster, UK, LA1 4YW \\ ${ }^{2}$ Physics Department, Sultan Qaboos University \\ Muscat, Oman, 123
}

E-mail: h.alhamrashdielancaster.ac.uk or hajir@squ.edu.om

KEYWORDS: Gamma detectors, Neutron detectors (thermal and fast), Lithium glass scintillators GS10, Plastic scintillator EJ-204, Particle identification methods.

\begin{tabular}{llr}
\multicolumn{2}{l}{ Contents } & $\mathbf{2}$ \\
$\mathbf{1}$ & Introduction & $\mathbf{2}$ \\
$\mathbf{2}$ & Pulse discrimination analysis & $\mathbf{4}$ \\
$\mathbf{3}$ & Experimental setup & $\mathbf{7}$ \\
$\mathbf{4}$ & Results & 7 \\
4.1 & Pulse height analysis method (PHA) & 9 \\
4.2 & Charge Comparison method (CCM) & 11 \\
& 4.3 & Pulse gradient analysis (PGA) \\
$\mathbf{5}$ & Discussion and conclusion & $\mathbf{1 2}$ \\
References & $\mathbf{1 4}$
\end{tabular}

ABSTRACT: Two radiation sensitive scintillators known for their dual sensitivity to neutron and gammaray fields are investigated for their pulse discrimination abilities; a lithium glass GS10 inorganic scintillator and a fast organic plastic scintillator EJ-204. Each of these scintillators are optically coupled with an 8x8-silicon photomultiplier array to act as a photodetector. Pulse height analysis, the charge comparison method and pulse gradient analysis have all been applied here on neutron and gamma-ray events generated by a Cf-252 source. The three discrimination methods were evaluated based on the figure of merit of the probability density plots generated. Within a GS10 crystal, it has been deduced that pulse height analysis and pulse gradient analysis possess greater abilities to discriminate between the two radiation fields compared to the charge comparison method with both showing a figure of merit of over one. The charge comparison method indicated a lower discrimination ability with a figure of merit around 0.3. When the EJ-204 detector was used, it was deduced that only pulse height analysis exhibits discrimination abilities with a figure of merit around 0.6, while the other two discrimination methods presented no distinction between the two radiation fields. 


\section{Introduction}

Applications in which mixed neutron and gamma-ray radiation fields are involved have gained steady interest through the years, especially in the areas of non-proliferation [1-3], radiography and tomography [4-8] and medical imaging [9]. Two approaches made it possible for these applications to succeed: scintillation materials sensitive to both radiation fields and multi-detector systems. The field of scintillation materials sensitive to both radiation fields has gone through significant evolvement since the 1950s. It started with the practical investigation of organic scintillators with dual sensitivity in the late 1950s [10-12] to organic plastic scintillators and glass scintillators in the 1960s [12-14] to semiconductor detectors in the 2000s $[15,16]$ and recently to the new era of elpasolite family scintillators and new generation scintillators $[17,18]$. Research on multi-detector systems aimed at investigating both radiation fields has exhibited a slow but steady increase through the years with the first reported multi-detector system described by Aryaeinejad and Spencer in 2004 in which side-byside lithium isotope loaded glass scintillators were used [19]. This was a handheld detection device with no imaging capabilities. In addition, the system was sensitive to background radiation with no direct means of collimation to eliminate background noise. Another dual neutron and gamma-ray imaging concept involves the use of organic and sometimes inorganic scintillator arrays arranged in parallel layers. Examples of this array-based design are the multi-layered electronic collimation based approach investigated by Polack et al. [20,21] and the multi-layered coded-aperture based approach investigated by Ayaz-Maierhafer et al.[22]. A noticeable drawback in these designs are the scintillators integrated with PMT units which can be bulky and heavy with the most utilised detectors in multiple detector/multi-layered designs being liquid organic scintillators (mainly EJ-309) and inorganic scintillators (usually $\mathrm{NaI}(\mathrm{Tl})$ and $\mathrm{CsI}(\mathrm{Tl})$ ) [20-22]. The next step forward from these instruments involves compact and fast scintillation materials that are sensitive to either or both radiation fields.

In this work, two scintillators with different properties are investigated for their abilities to be integrated in a highly compact, very fast (real-time scale), dual particle, multi-layered imaging system. These two scintillation material are natural Lithium glass GS10 from Scintacor [23] (6.6\% total lithium content of which $7.59 \%$ Li-6 and $92.41 \%$ Li-7) and fast plastic scintillator EJ-204 from Eljen Technology [24] (commercial equivalents BC-404 and NE-104). In terms of neutron detection abilities, the GS10 detector possesses a thermal neutron capture efficiency of $90 \%$ at a $20 \mathrm{~mm}$ thickness. In addition, GS10 possesses distinctively higher fast neutron backscattering and forward scattering abilities compared to other scintillation detectors including CLYC from the elpasolite scintillators family and EJ-309 from the liquid scintillator category [25]. Moreover, being a glass-based scintillator, GS10 boasts enhanced durability in harsh radiation environments. Among other commercial glass scintillators, GS10 possess moderate lithium content, which allows GS10 to be effectively more sensitive to both neutrons and gamma rays compared to other lithium glass detectors in the same commercial category [23]. On the other hand, EJ-204 is a multipurpose Polyvinyl toluene (PVT) organic plastic scintillator with $5.15 \times 1022$ hydrogen atoms $/ \mathrm{cm} 3$. It is characterised by a high-speed response with a $2.2 \mathrm{~ns}$ pulse width and $1.8 \mathrm{~ns}$ decay time. In addition, EJ-204 has the highest scintillation efficiency among other plastic scintillators with 10,400 photons/MeV e- [24]. This collection of desirable characteristics ensures that EJ-204 is strongly favoured within neutron scattering applications, especially when compact designs and a fast Time of Flight (TOF) are major requirements [26].

\section{Pulse discrimination analysis}

Pulse discrimination analysis was reported in the literature as early as the 1950s [10,11]. The most common technique of pulse discrimination analysis is known as Pulse Shape Discrimination (PSD), which takes advantage of the differences in the time constants of the scintillation pulses generated by gammas and neutrons. In inorganic scintillators, absorption of radiation energy excites electrons within the energy levels of the crystal or glass lattice. Impurities are key components in the scintillation process of inorganic scintillators via which self-absorption of emitted light is minimised [27], although pure 
crystal cases such as diamond are exceptions to this [27]. Commonly, within inorganic impurityactivated scintillators such as $\mathrm{Ce}$-activated glass detectors and Tl-activated alkali detectors, the energy bands have impurity sites in which electrons might emigrate and eventually de-excite resulting in the emission of scintillation light [27]. A discussion on the difference in the response of inorganic scintillators including glass scintillators is discussed widely in literature [27-30]. The outcome of these discussions suggest that different ionising radiations have different energy loss rates and therefore the scintillation process is different for different radiation fields. Similarly, organic scintillators are widely employed due to the nature of the scintillation process adopted in them [11,31, 32]. As described by Brooks et al. [33], the innate molecular characteristics of organic scintillators is responsible for the luminescence process. Within the $\pi$-electronic energy levels of the unsaturated aromatic or heterocyclic molecules, there are the singlet states (So, S1, S2 ...) and triplet states (To, T1, T2 ...). Excitation or ionisation of electrons due to energy absorption to singlet states and then de-excitation to singlet states results in the prompt emission of scintillation light known as fluorescence. Fluorescence is characterised by a short lifetime between $10 \mathrm{~ns}$ and $1 \mathrm{~ns}$ (S1 level de-excitation). On the other hand, excitation from singlet states and de-excitation from triplet states results in a delayed and slower emission of scintillation known as phosphorescence. This type of emission is characterised by a longer wavelength and decay time and appears to be the predominant recombination process ( $75 \%$ of the time) [27]. Scintillation luminescence varies with the energy loss rate in organic scintillators including plastic scintillators. Therefore, ionising radiations have varying energy loss rates and the scintillation process is thus different for each radiation field type [27]. More specifically protons generated via neutron scattering reactions in organic scintillators result in a higher number of triplet state based phosphorescence photons and more excited electrons compared to gamma rays. This gives neutrongenerated pulses a longer decay time compared to other radiation fields [34]. There are two main PSD approaches for scintillators with PSD potentials: the analog approach and the digital approach. Table 1 shows the most common PSD techniques based on these two approaches in literature.

\section{Table 1: Common PSD techniques in literature.}

\begin{tabular}{ll}
\hline Analog approach & Digital approach \\
\hline Pulse height analysis & Pulse height analysis \\
Zero-crossing & Zero crossing \\
Charge comparison & Charge comparison \\
& Optimal linear filter \\
& Pulse gradient analysis \\
& Frequency gradient analysis \\
& Artificial neural network \\
& Triangular filter \\
& Power spectrum analysis \\
\hline
\end{tabular}

The Pulse Height Analysis method (PHA) is based on comparing the total scintillation light generated by the radiation field. It is highly recommended for scintillation detectors in which the scintillation photon generation process shows no major difference when either heavy charged particle ionisation events and electron ionisation events occur [35]. In the zero-crossing technique, the pulse is converted into a bipolar pulse with the time interval between the starting point of the pulse and the point at which the pulse crosses zero measured. The Charge Comparison Method (CCM) is a well-established method 
in the field of PSD. Applied in both analog and digital approaches, this method is based on comparing the integrals of the charge over two time intervals commonly known as the short integral and the long integral $[12,34]$. The time intervals are selected based on the pulse characteristics of the detector under investigation. This method has been shown to be very successful within organic scintillators where a long decay time is commonly observed for scintillation photons generated by the ionisation caused by heavily charged particles.

Digital methods such as the optimal linear filter technique require the use of model data and labelled data template sets of expected neutron gamma pulse shapes. Normalised pulses are compared to the template sets with gamma-ray and neutron pulses separated [36]. The optimal linear filter process is lengthy and requires a substantial training data set. In addition, this method might not be applicable for very fast pulses where accurate digitisation needs an ultra-high speed acquiring system. Pure digital PSD techniques thrived after advances in semiconductor technologies, surface mount electrical circuit components and analog to digital converter circuits. Data acquiring systems and field programmable gate arrays (FPGA's) in particular allowed fast digitisation and analysis of data, which greatly enhanced the performance of these techniques. Within the field of organic scintillation detectors, these techniques have proven to be highly efficient [37-41]. Pulse gradient analysis (PGA), successfully first discussed by D'Mellow et al. [37], compares the gradient of a normalised pulse at a fixed point on the peak (known as the first integral) to a second gradient at a second point located at a fixed time interval from the first integral, and known as the second integral. Another digitally based method is Frequency Gradient Analysis (FGA) [39] where the pulse is transformed into the frequency domain. When a discrete Fourier transformation is utilised in the conversion method, PSD is performed by comparing the point at zero frequency to the point at the first frequency component of the discrete Fourier transform. A more comprehensive method is the artificial neural network method which compares the results to data sets [41]; the system being trained artificially or compared to previously approved data sets. However, the method requires substantial data sets, which might not be applicable to all scintillation detectors. The triangular filter method transforms the pulses using passive and active triangular shaping circuits with pulses compared to each other [42]. Power spectrum analysis is very similar to frequency gradient analysis where the pulse is transferred to the frequency domain [43].

To the knowledge of the authors, GS10 and EJ-204 scintillators have never been directly investigated for their neutron and gamma-ray discrimination abilities and with the rise of dual particle detection applications along with the previously outlined characteristics of these two detectors, this work offers a preparatory foundation for applications involving these two scintillation materials. The discrimination method criteria should fit the application intended in this research where a fast and low processing power digital technique is required for a dual-particle multi-layered design in which fast pulses from GS10 and EJ-204 are investigated. Three of the previously outlined methods follow these criteria, the PHA method, the CCM and the PGA method.

\section{Experimental setup}

Within the experiment, the GS10 crystal used had dimensions of $27 \mathrm{~mm} \times 27 \mathrm{~mm} \times 15 \mathrm{~mm}$, whereas the EJ-204 plastic scintillator had dimensions of $27 \mathrm{~mm}$ x $27 \mathrm{~mm}$ x $20 \mathrm{~mm}$. A Cf-252 source was utilised throughout the experiment to generate the required neutron particles and gamma-ray photons. Cf-252 is a spontaneous fission source with a half-life of 965 days and a branching ratio of 3.09\% [44]. The energy spectrum of Cf-252 fission neutrons follow the Watt spectrum fit with an average neutron energy of $2.1 \mathrm{MeV}$, most probable energy of $0.7 \mathrm{MeV}$ and a yield of 3.759 per spontaneous fission decay [45]. The average energy of the associated gamma-ray component is $0.8 \mathrm{MeV}$ with average prompt fission multiplicity of 8.3 gamma-ray photons per fission event [46]. The scintillation detectors assembly arranges the scintillators in series, with the GS10 scintillator located $3 \mathrm{~cm}$ behind the EJ-204 scintillator. The photodetector utilised in the experiment is the $8 \times 8$ SiPM (ArrayJ-30035-64P-PCB 8x8 SiPM, 
SensL, Cork, Ireland [28]). These detection arrays are placed in optical contact with the scintillators within each layer, and are mounted on ARRAYJ-BOB3-64P (SensL) breakout boards. Each SiPM pixel has a designated simple current-to-voltage converter consisting of a $47 \Omega$ resistor in series with the diode. A dual benchtop power supply was used to provide $29.4 \mathrm{~V}$ of reverse bias which was applied across the resistor and diode. The assembly was placed $10 \mathrm{~cm}$ way from the water tank housing the Cf252 source. Figure 1 illustrates the arrangement of the source and the detectors assembly in the experiment.

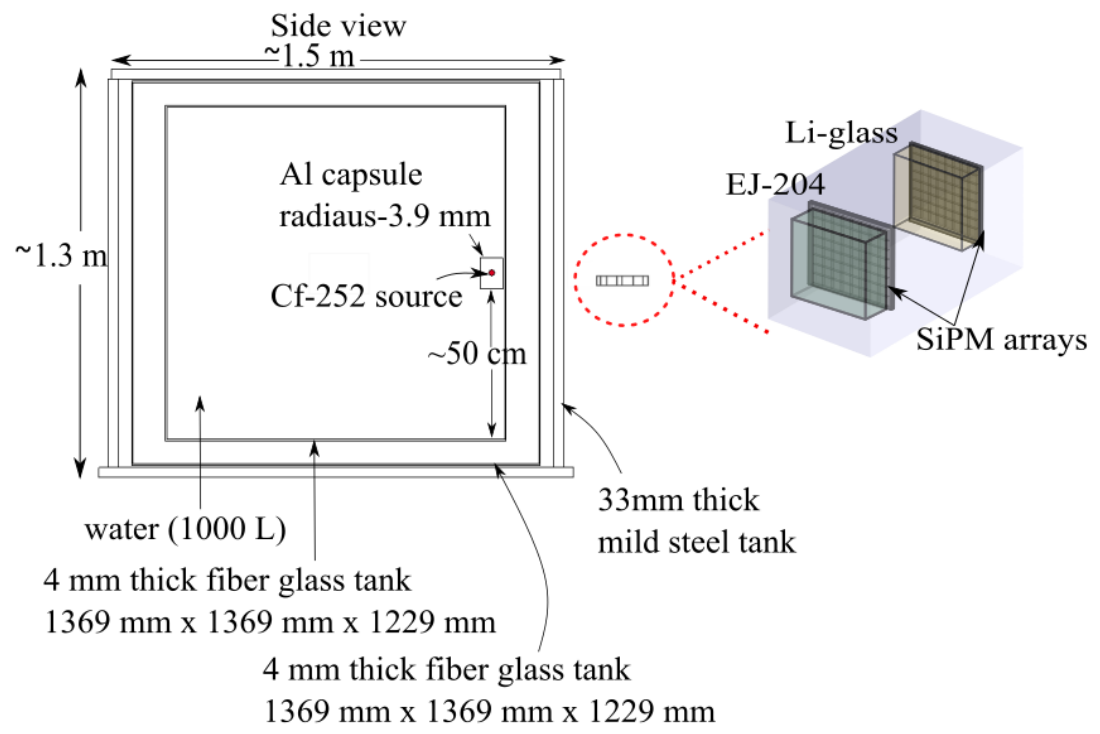

Figure 1: The $\mathbf{C f - 2 5 2}$ source housing used in studying the response of the scintillation detectors along with the scintillation detectors assembly located in front of the tank. GS10 scintillator is located $3 \mathrm{~cm}$ behind EJ-204 scintillator.

In general, the response of the scintillators and SiPM arrays depends on a number of geometrical factors (such as the size and the shape of the scintillators, the optical coupling and the SiPM array geometry), and their physical characteristics (the dark pulses and electrical noise for this photodetector at $\sim 1.1 \mu \mathrm{A}$ at $29.4 \mathrm{~V}$ ). A simple integration circuit was created with a $1 \mathrm{nF}$ capacitor across the ports where the pulses are acquired using an Agilent 54845A Infinium Oscilloscope with a sampling speed of $8 \mathrm{GSa} / \mathrm{s}$ and bandwidth of $1.5 \mathrm{GHz}$. Two dedicated MATLAB® programs (described in [47]) were used while conducting the experiment and subsequent analysis of the pulses. The first program mediated the pulses from the oscilloscope to the PC while the second program analysed the data. Over 10,000 pulses from the GS10 and EJ-204 scintillators were acquired and analysed in this work. Figure 2 shows a block diagram of the experimental setup. The pulses are sent to the acquiring system, the Agilent 54845A Infinium Oscilloscope, via ribbon cables. The acquired data are then sent via an Ethernet cable to the control area. 


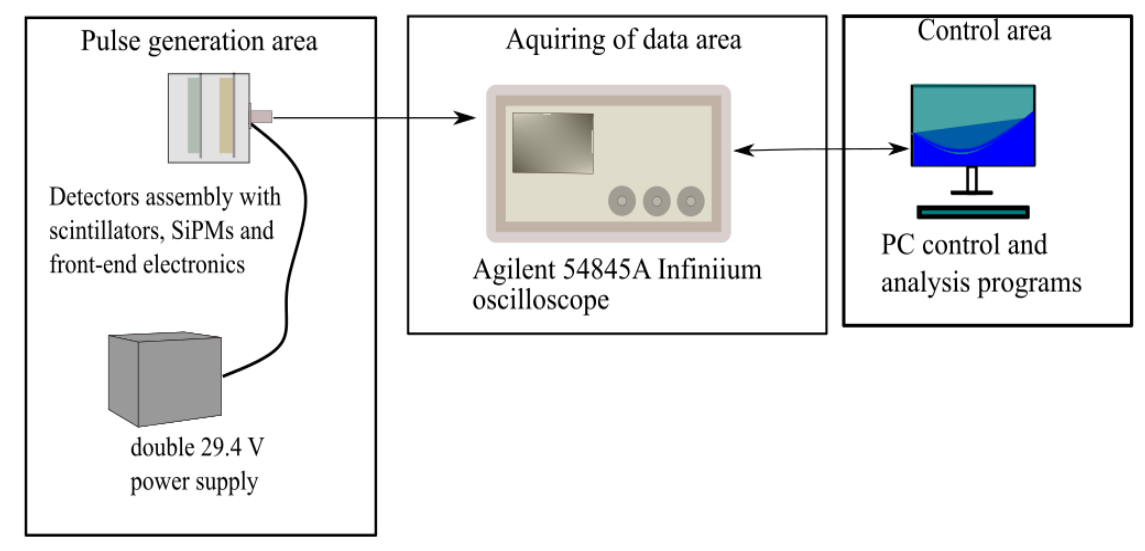

Figure 2: The main components in the experimental setup; (L-R): The pulse generation area with EJ-204 and GS10 scintillators and SiPM arrays, the data acquisition area comprised of Infinium Oscilloscope and finally the PC control area.

The raw pulses acquired from the GS10 glass varied in amplitude and shape. Figure 3 shows examples of neutron and gamma-ray pulses directly generated from GS10 and visually digitised by the Agilent 54845A Infinium Oscilloscope.

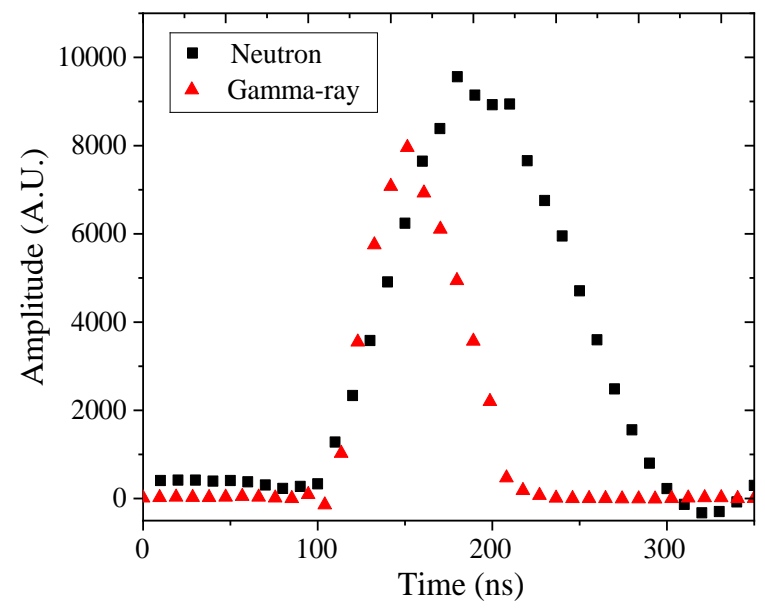

Figure 3. Examples of Cf-252 neutron and gamma-ray raw pulses acquired directly from the GS10 scintillator.

EJ-204 is characterised by its fast response and hence short scintillation pulse width, estimated to be $2.2 \mathrm{~ns}$ [24]. The high sampling speed of the Agilent 54845A Infiniium Oscilloscope allows the digitising of EJ-204 pulses with a rate of 1 point every $~ 1 / 4$ ns and hence the plotting and acquiring of the pulses as shown in Figure 4. However, the finite sampling rate of the acquisition system will affect the CCM and PGA methods where higher resolution representation of the pulses is essential for the success of these methods. 


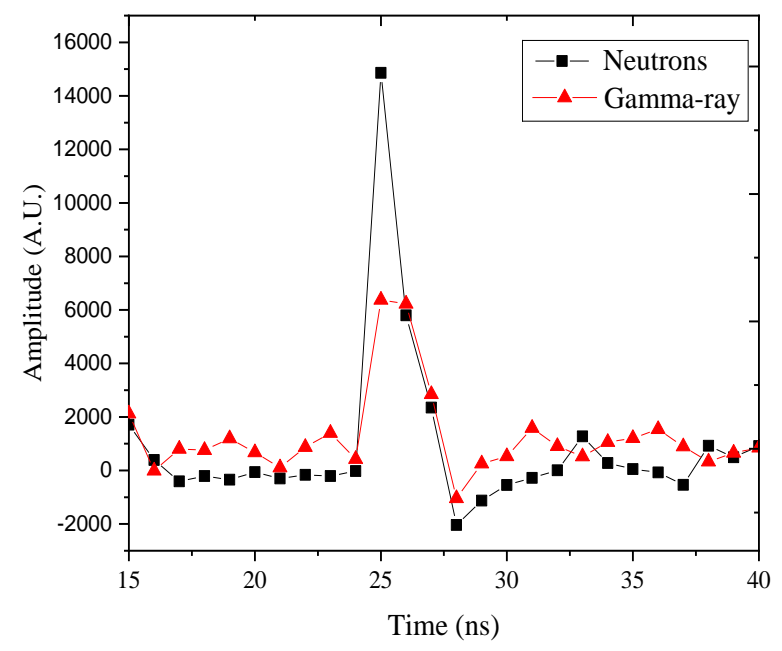

Figure 4. Examples of Cf-252 neutron and gamma-ray photon neutron and gammaray raw pulses acquired directly from EJ-204 scintillation detector (the line joining the data points are added for clarity).

\section{Results}

\subsection{Pulse height analysis method (PHA)}

Both neutron and gamma-ray events result in the generation of scintillation light in GS10 and EJ204. However, the integrated intensity of scintillation photons per unit length depends on the charge of the secondary particles generating primary excitations in the scintillation material [27, 34]. Therefore, scintillation light generated from gamma-ray photon interaction in these two scintillators is usually less than the scintillation light generated by neutron interaction. As a result, direct measurement and comparison of the magnitude of generated electrical pulses allows the discrimination of gamma-ray events from neutron events. Figure 5 shows the relative frequency of events representing the normalised number of pulses as function of integrated charges (i.e. pulse height) for gamma-ray photons and neutrons within a GS10 scintillator. The figure shows how a Gaussian fit of neutron generated pulses have a higher pulse amplitude compared to the Gaussian fit to the gamma-ray pulses, allowing a clear and a comfortable discrimination between events generated by these two radiation fields. Based on the results in Figure 5, neutron generated pulses have an average pulse height of (94000 \pm 1000$)$ ADC units with a Full Width at Half Maximum $(F W H M)=(21000 \pm 3000)$ ADC units and a spectrum resolution of 0.49 , whereas gamma-ray generated pulses have an average pulse height of $(47620 \pm 20)$ ADC units with a spread of FWHM $=(3610 \pm 40)$ ADC units and a spectrum resolution of 0.076 . This method can be implemented directly within the readout circuit of the scintillation detector by setting a voltage threshold level using the pulse height peak level and the associated confidence level for the two events. 


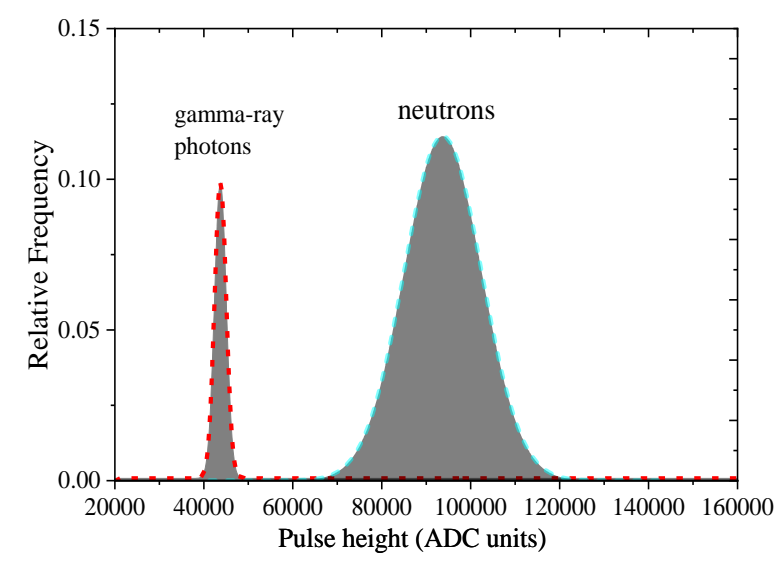

Figure 5. Relative frequency of events as function of pulse height for neutron and gammaray pulses from Cf-252 source in a GS10 detector. Gamma-ray photons generated pulses (left) and neutron generated pulses (right) are distinctly separated.

For the EJ-204 scintillation detector, the total integrated charge generated by neutron events and gamma-ray photons are measured and normalised to the total number of pulses. The relative frequency of pulses are presented as a function of pulse height in a probability density plot in Figure 6 . The figure shows two clear peaks for neutron and gamma-ray photons, however, intersection between the pulses is clear in the figure. This indicates an ambiguity of $\sim 8 \%$ of neutron events and $\sim 15 \%$ of gamma-ray events. This can lead to significant loss in efficiency for scattering based imaging systems. In addition, the results in Figure 5 show that neutron generated pulses have an average pulse height of $(20000 \pm$ 800) ADC units with FWHM $=(6100 \pm 2000) \mathrm{ADC}$ units and a spectrum resolution of 0.30 , whereas gamma-ray generated pulses have an average pulse height of $(11000 \pm 2000)$ ADC units with FWHM $=(10000 \pm 4000) \mathrm{ADC}$ units and a spectrum resolution of 0.9. The wide spread in the relative frequency counts of pulse heights for EJ-204 resulted on high uncertainty in the average pulse height values and FWHM values for both radiation fields. 


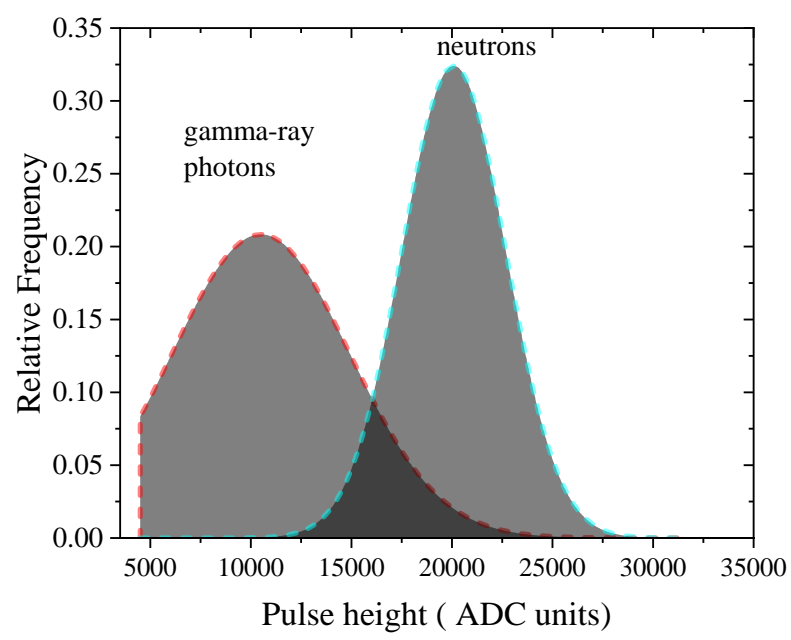

Figure 6. Relative frequency of events as function of pulse height for neutron and gammaray pulses from Cf-252 source in a EJ-204 detector. Gamma-ray photons generated pulses (left) and neutron generated pulses (right) are intersecting over an area of $\mathbf{1 0 0 0 0}$ ADC units.

\subsection{Charge Comparison method (CCM)}

The charge comparison method evaluates the integrated charges in a pulse over two periods of time commonly known as the long integral and the short integral. In here, the long integral corresponds to the area of the entire pulse whereas the short integral corresponds to the area covering part of the pulse commonly set at an optimised sample time on the decaying part of pulse [42]. For a GS10 detector, the charge comparison method was applied to the acquired pulses with a short integral set at $50 \mathrm{~ns}$ after the peak amplitude. A scatter plot of the short integral against the long integral was created as illustrated in Figure 7. The figure shows a cross-reference area where the events overlap. However, the majority of gamma-ray events are located below the diagonal line crossing the zero reference point in the figure.

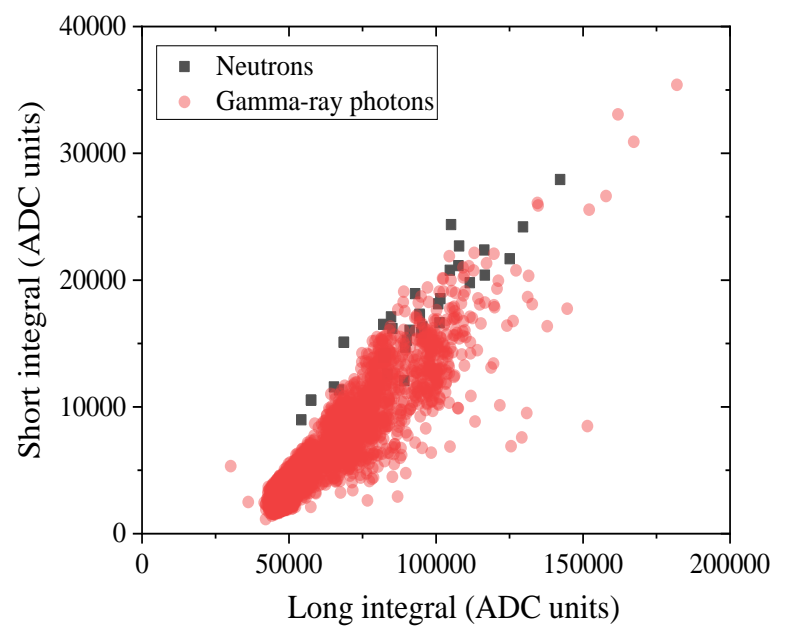

Figure 7. A scatter plot of the acquired pulses short integral in the y-axis and long integral in the $\mathrm{x}$-axis from $\mathrm{Cf}-252$ source in GS10.

The pulses in Figure 7 were normalised to the total number of events in the corresponding radiation group and a probability distribution plot with the relative frequency against the ratio of short integral to long integral was generated. Figure 8 shows the two Gaussian fits of the probability distribution plot of 
the total events. The neutron events have an average short integral to long integral ratio of $(0.35 \pm 0.01)$ with a spread of $\sigma=(0.11 \pm 0.07)$. The gamma-ray events have an average short integral to long integral ratio of $(0.80 \pm 0.06)$ with spread $\sigma=(0.45 \pm 0.07)$. Events clearly overlap in the probability distribution plot suggesting low discrimination efficiency.

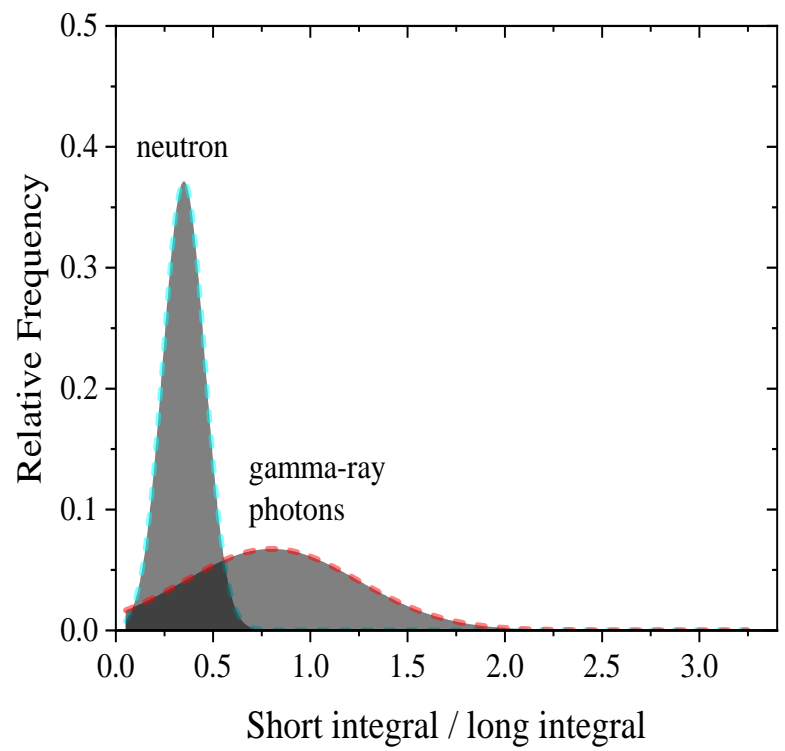

Figure 8. Relative frequency of events against short integral to long integral ratio of all events generated by gamma-ray photons (centre left) and neutrons (far left) from a Cf-252 source in a GS10 detector.

Attempts were made to generate a discrimination platform for an EJ-204 detector using charge comparison discrimination methods. However, as shown in Figure 9 there were no clear discrimination levels between the two events with complete overlap between the neutron and gamma-ray events probability distribution functions.

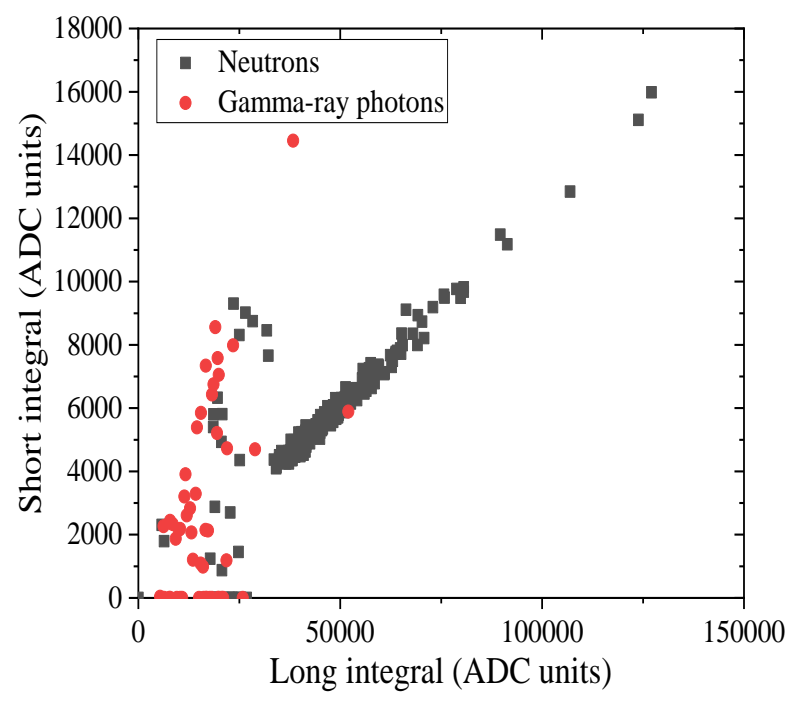

Figure 9. A scatter plot of the acquired pulses short integral in the y-axis and long integral in the $\mathrm{x}$-axis from Cf-252 source in EJ-204. 


\subsection{Pulse gradient analysis (PGA)}

The PGA method uses the differences in the time domain over the decay interval of the pulses generated by gamma-ray photons and neutrons. The main advantage of this method is that it compares parameters that are sensitive to any changes in the production mechanism of scintillation photons allowing subtle deduction of pulse variations. For a GS10 crystal, the time constant at which the sample amplitude was investigated against peak amplitude was optimised at $50 \mathrm{~ns}$ after the peak amplitude. Figure 10 illustrates the sample amplitude as function of the peak amplitude for combined neutron and gamma-ray events.

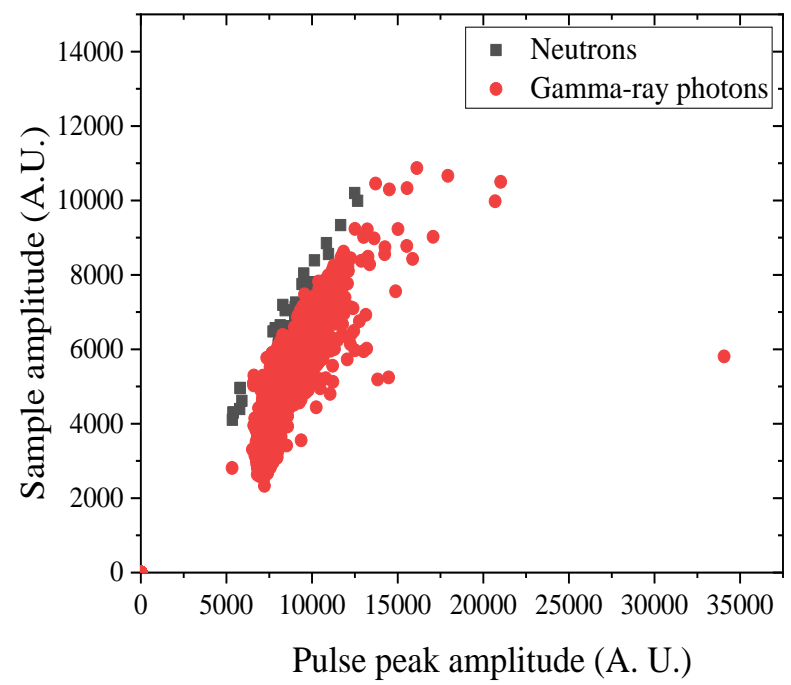

Figure 10. A scatter plot of the acquired data from $\mathrm{Cf}-252$ source in a GS10 scintillator. The sample amplitude of the pulses $50 \mathrm{~ns}$ after the peak amplitude is plotted against the peak amplitude.

The pulses in figure 10 were normalised to the total number of pulses in the corresponding radiation type and a probability distribution plot with the relative frequency against the ratio of sample amplitude to peak amplitude was generated. Figure 11 shows two Gaussian fits of the probability distribution plot of neutron events and gamma-ray events. The neutron events have an average PGA ratio of $(0.788 \pm$ $0.001)$ with a spread of $\sigma=(0.018 \pm 0.001)$. The gamma-ray events had an average PGA ratio of $(0.510$ $\pm 0.004)$ with a spread of $\sigma=(0.085 \pm 0.005)$. 


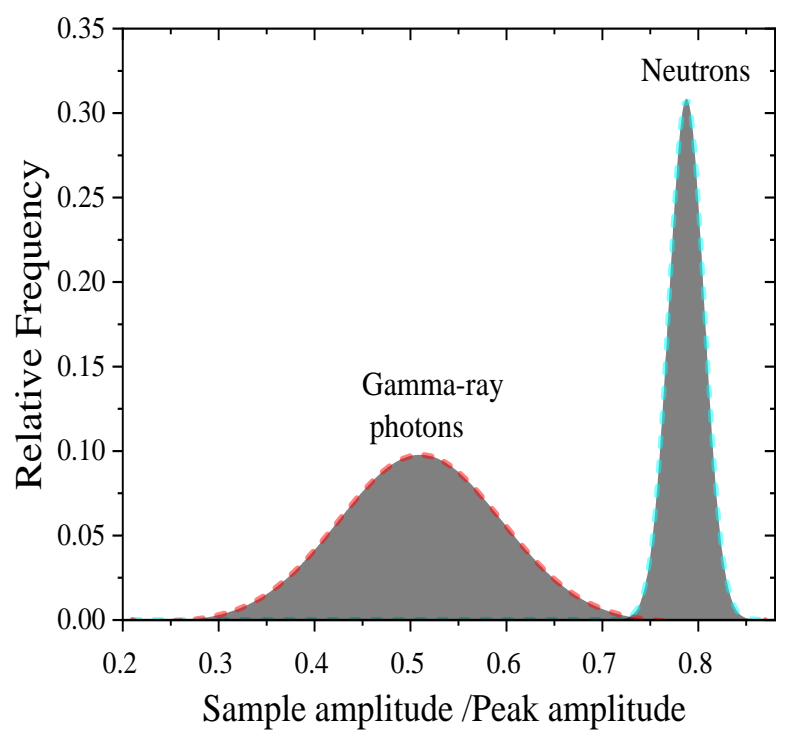

Figure 11: Relative frequency of events as function of pulse height generated by gamma-ray photons (centre) and neutrons (left) from a Cf-252 source in GS10 detector.

For EJ-204 the sample amplitude was attempted at different peak lag times. However, there was no clear discrimination level between the two events and they completely overlapped. This can be clearly seen in Figure 12 where a scatter plot of the sample amplitude against peak amplitude at $1 \mathrm{~ns}$ after the peak amplitude show complete overlapping of events from the two radiation fields.

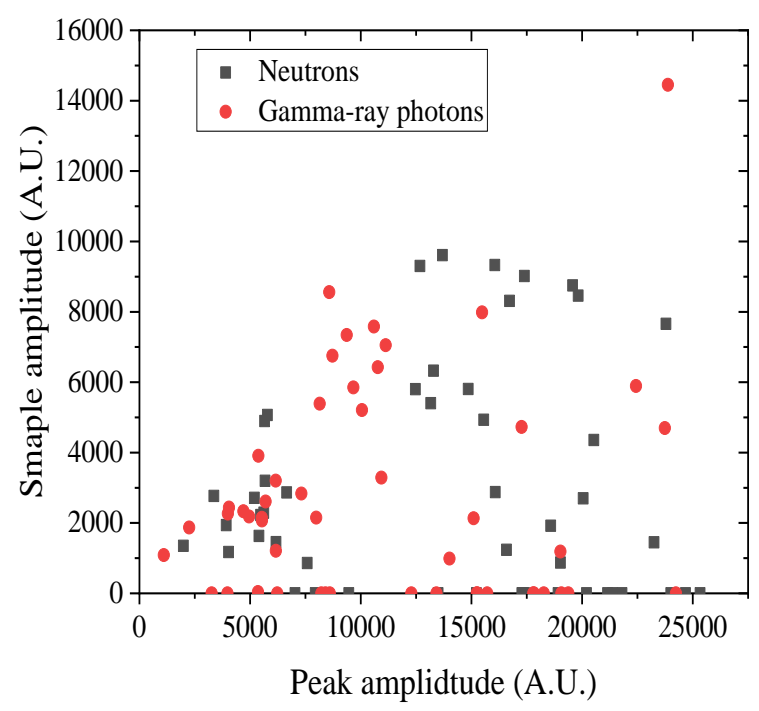

Figure 12. A scatter plot of the acquired data from $\mathrm{Cf}-252$ source in EJ-204 detector. The sample amplitude of the pulse is plotted against the amplitude of the peak amplitude with complete overlap of events in the figure area.

\section{Discussion and conclusion}

Three different pulse discrimination methods, PHA, CCM and PGA, were used to discriminate neutron and gamma-ray events in GS10 and EJ-204 scintillation detectors. One common technique 
applied in evaluating and comparing the quality of discrimination methods is the figure of merit, FOM. Commonly, the FOM is obtained from the probability distribution plot of the discrimination method under review. The FOM in a probability distribution plot can be expressed as [28]

$$
\mathrm{F} \mathrm{OM}=\frac{\left|\mathrm{Peak}_{\gamma}-\mathrm{Peak}_{\mathrm{n}}\right|}{\mathrm{FWHM}_{\gamma}+\mathrm{FWHM}_{\mathrm{n}}}
$$

Where Peak $\mathrm{k}_{\gamma}$ is the gamma-ray events peak position, $\mathrm{Peak}_{\mathrm{n}}$ is neutron events peak position, $\mathrm{FWHM}_{\gamma}$ is gamma-rays peak full width at half maximum value and $\mathrm{FWHM}_{\mathrm{n}}$ neutrons peak full width at half maximum value. For example, in Figure 11, Peak $_{\gamma}=0.51$, Peak $_{\mathrm{n}}=0.79, \mathrm{FWHM}_{\gamma}=0.2$ and $\mathrm{FWHM}_{\mathrm{n}}=$ 0.04 , based on equation (1), FOM in this case is 1.1. The statistics characterising three discrimination methods applied in this work along with FOM values based on figures 5,6, 8 and 10 are summarised in Table 2.

Table 2: Summary of the average values and the FOMs of the three pulse discrimination methods applied on GS10 and EJ-204 neutron and gamma-ray pulses from Cf-252 source.

\begin{tabular}{|l|c|c|l|l|l|l|}
\hline $\begin{array}{l}\text { Pulse } \\
\text { discriminatio } \\
\text { n method }\end{array}$ & $\begin{array}{l}\text { Neutrons } \\
\text { average }\end{array}$ & $\begin{array}{l}\text { Gamma-ray } \\
\text { average }\end{array}$ & FOM & $\begin{array}{c}\text { Neutrons } \\
\text { average }\end{array}$ & $\begin{array}{c}\text { Gamma- } \\
\text { ray } \\
\text { average }\end{array}$ & FOM \\
\hline $\begin{array}{l}\text { PHA (ADC } \\
\text { units for } \\
\text { average } \\
\text { values) }\end{array}$ & $(92 \pm 2) \times 10^{5}$ & $(53 \pm 1) \times 10^{5}$ & 1.00 & $(22 \pm 4) \times 10^{5}$ & $(13 \pm 6) \times 10^{5}$ & 0.682 \\
$\begin{array}{l}\text { short integral } \\
\text { to long } \\
\text { integral ratio }\end{array}$ & $0.35 \pm 0.10$ & $1.1 \pm 0.5$ & 0.383 & $1.000 \pm 0.002$ & $1.00 \pm 0.001$ & NA \\
PGA ratio & $3.1 \pm 0.9$ & $2.05 \pm 0.05$ & 1.10 & $0.21 \pm 0.21$ & $0.30 \pm 0.21$ & NA \\
\hline
\end{tabular}

In the case of the GS10 scintillation detector, neutron pulses have a distinctly higher pulse amplitude compared to gamma-ray pulses. Similarly, PGA analysis on GS10 shows that neutron pulses generally have a higher sample amplitude to peak amplitude ratio (defined in Table 2 as PGA ratio). Reflecting on the FOM values in Table 2, neutron and gamma-ray pulses discrimination is applicable for a GS10 scintillation detector via the PHA and PGA method with both showing FOM values around 1. In contrast, the charge comparison method shows poor discrimination abilities between neutron and gamma-ray pulses in a GS10 scintillation detector with a FOM value of around 0.383. In the case of the EJ-204 scintillation detector, neutron pulses acquire a higher pulse amplitude compared to gamma-ray pulses with a FOM equal to 0.682 suggesting the possibility of successful PHA in a mixed radiation field measurement. However, loss in counts from both radiation fields will occur as a result. Both the CCM and PGA methods suggests pulse shape discrimination is not applicable for EJ-204 in this work. One major reason for this outcome is the poor presentation of EJ-204 pulses in the acquiring system (i.e. the oscilloscope). The pulses generated by EJ-204 are very fast with a $2.2 \mathrm{~ns}$ pulse width. The oscilloscope digitises the pulse with sampling speed of $8 \mathrm{GSa} / \mathrm{s}$, a sampling speed that is sufficiently fast enough to acquire the pulse. However, a higher sampling speed is required to acquire more samples per pulse allowing higher accuracy in recreating the shape of the pulses later in the analysis stage. There are a number of oscilloscopes with a higher sampling rate than the one utilised within this study. However, the aim within this work is to utilise a sampling speed comparable to the best data acquisition 
systems currently available for integration in portable radiation detection systems such as Field Programmable Gate Arrays (FPGAs) and Data Acquisition (DAQ) systems. In addition, this work is intended to create a platform for further investigation in the pulse discrimination abilities of two detectors, which possess great potential in the field of dual-particles detection but yet are currently understudied in literature.

\section{References}

1. $\quad$ Polack, J.K., et al., Image Reconstruction Using a Three-Plane, Dual-Particle Imager for Standoff Detection of Special Nuclear Material, in 2012 IEEENuclear Science Symposium and Medical Imaging Conference Record, B. Yu, Editor. 2012. p. 118-121.

2. Ayaz-Maierhafer, B., et al., Transmission and signal loss in mask designs for a dual neutron and gamma imager applied to mobile standoff detection. Nuclear Instruments \& Methods in Physics Research Section a-Accelerators Spectrometers Detectors and Associated Equipment, 2013. 712: p. 1-8.

3. Cester, D., et al., A novel detector assembly for detecting thermal neutrons, fast neutrons and gamma rays. Nuclear Instruments and Methods in Physics Research Section A: Accelerators, Spectrometers, Detectors and Associated Equipment, 2016. 830: p. 191-196.

4. Devolpi, A. and E.A. Rhodes, Neutron and gamma-ray tomographic imaging of LMFBR SAREF-program safety-test fuel assemblies. MATER EVAL, 1982. V 40(N 12): p. 12731279 .

5. Chaouki, J., F. Larachi, and M.P. Duduković, Noninvasive Tomographic and Velocimetric Monitoring of Multiphase Flows. Industrial and Engineering Chemistry Research, 1997. 36(11): p. 4476-4503.

6. Gozani, T., The role of neutron based inspection techniques in the post 9/11/01 era. Nuclear Instruments and Methods in Physics Research, Section B: Beam Interactions with Materials and Atoms, 2004. 213: p. 460-463.

7. Kapadia, A.J., et al. GEANT4 simulation of an NSECT system for iron overload detection. in IEEE Nuclear Science Symposium Conference Record. 2007.

8. Sowerby, B.D., et al. Recent developments in fast neutron radiography for the interrogation of air cargo containers. in International Topical Meeting on Nuclear Research Applications and Utilization of Accelerators. 2009.

9. Biegun, A.K., et al., Time-of-flight neutron rejection to improve prompt gamma imaging for proton range verification: a simulation study. Physics in Medicine and Biology, 2012. 57(20): p. 6429-6444.

10. Owen, R.B., The Decay Times of Organic Scintillators and Their Application to the Discrimination Between Particles of Differing Specific Ionization. IRE Transactions on Nuclear Science, 1958. 5(3): p. 198-201.

11. Brooks, F.D., A scintillation counter with neutron and gamma-ray discriminators. Nuclear Instruments and Methods, 1959. 4(3): p. 151-163.

12. Brooks, F.D., R.W. Pringle, and B.L. Funt, Pulse Shape Discrimination in a Plastic Scintillator. IRE Transactions on Nuclear Science, 1960. NS-7(2-3): p. 35-38.

13. Coceva, C., Pulse-shape discrimination with a glass scintillator. Nuclear Instruments and Methods, 1963. 21(C): p. 93-96.

14. Smith, D.L., R.G. Polk, and T.G. Miller, Measurement of the response of several organic scintillators to electrons, protons and deuterons. Nuclear Instruments and Methods, 1968. 64(2): p. 157-166.

15. Martín-Martín, A., et al., Evaluation of CdZnTe as neutron detector around medical accelerators. Radiation Protection Dosimetry, 2009. 133(4): p. 193-199.

16. Tupitsyn, E., et al., Single crystal of LiInSe $e_{2}$ semiconductor for neutron detector. Applied Physics Letters, 2012. 101(20).

17. Glodo, J., et al. $\mathrm{Cs}_{2} \mathrm{LiYCl}_{6}$ : Ce Neutron gamma detection system. in 2007 IEEE Nuclear Science Symposium Conference Record. 2007. Honolulu, HI, USA. 
18. Glodo, J., et al., Selected Properties of Cs2LiYCl6, Cs2LiLaCl6, and Cs2LiLaYBr6 Scintillators. Ieee Transactions on Nuclear Science, 2011. 58(1): p. 333-338.

19. Aryaeinejad, R. and D.F. Spencer, Pocket dual neutron/gamma radiation detector. IEEE Transactions on Nuclear Science, 2004. 51(4): p. 1667-1671.

20. Polack, J.K., et al. Dual-particle imager for standoff detection of special nuclear material. in 2011 IEEE Nuclear Science Symposium Conference Record. 2011. Valencia, Spain.

21. Poitrasson-Riviere, A., et al., Dual-particle imaging system based on simultaneous detection of photon and neutron collision events. Nuclear Instruments \& Methods in Physics Research Section a-Accelerators Spectrometers Detectors and Associated Equipment, 2014. 760: p. 4045.

22. Ayaz-Maierhafer, B., et al., Angular resolution study of a combined gamma-neutron coded aperture imager for standoff detection. Nuclear Instruments and Methods in Physics Research Section A: Accelerators, Spectrometers, Detectors and Associated Equipment, 2013. 712: p. 120-125.

23. 6-lithium glass bespoke to your application. [cited 2019 03/12/2019]; Available from: https://scintacor.com/products/6-lithium-glass/.

24. General Purpose Plastic Scintillators EJ-200, EJ-204, EJ-208, EJ-212. [cited 2019 25/07/2019]; Available from: https://eljentechnology.com/images/products/data_sheets/EJ200 EJ-204 EJ-208 EJ-212.pdf.

25. Al Hamrashdi, H., D. Cheneler, and S.D. Monk, Material optimization in dual particle detectors by comparing advanced scintillating materials using two Monte Carlo codes. Nuclear Instruments and Methods in Physics Research Section A: Accelerators, Spectrometers, Detectors and Associated Equipment, 2017. 869(Supplement C): p. 163-171.

26. Mascarenhas, N., et al. Development of a neutron scatter camera for fission neutrons. in IEEE Nuclear Science Symposium Conference Record. 2007. San Diego, CA, USA.

27. Birks, J.B., The theory and practice of scintillation counting. 1964: Pergamon Press, Macmillan, New York.

28. Winyard, R.A., J.E. Lutkin, and G.W. McBeth, Pulse shape discrimination in inorganic and organic scintillators. I. Nuclear Instruments and Methods, 1971. 95(1): p. 141-153.

29. Ranucci, G., An analytical approach to the evaluation of the pulse shape discrimination properties of scintillators. Nuclear Instruments and Methods in Physics Research Section A: Accelerators, Spectrometers, Detectors and Associated Equipment, 1995. 354(2): p. 389-399.

30. Spowart, A.R., Energy transfer in cerium-activated silicate glasses. Journal of Physics C: Solid State Physics, 1979. 12(16): p. 3369-3374.

31. Phillips, H.B. and R.K. Swank, Measurements of Scintillation Lifetimes. Review of Scientific Instruments, 1953. 24(8): p. 611-616.

32. Wright, G.T., Scintillation decay Times of organic crystals. Proceedings of the Physical Society. Section B, 1956. 69(3): p. 358-372.

33. Brooks, F.D., Development of organic scintillators. Nuclear Instruments and Methods, 1979. 162(1): p. 477-505.

34. Brooks, F.D., Development of organic scintillators. Nuclear Instruments and Methods, 1979. 162(1-3): p. 477-505.

35. Emmerich, W.S., A fast neutron scintillator. Review of Scientific Instruments, 1954. 25(1): p. 69-70.

36. Gatti, E. and F.D. Martini, A new linear method of discrimination between elementary particles in scintillation counters. 1962, International Atomic Energy Agency (IAEA): Brueder Rosenbaum.

37. D'Mellow, B., et al., Digital discrimination of neutrons and $\gamma$-rays in liquid scintillators using pulse gradient analysis. Nuclear Instruments and Methods in Physics Research, Section A: Accelerators, Spectrometers, Detectors and Associated Equipment, 2007. 578(1): p. 191-197.

38. Aspinall, M.D., et al., The empirical characterization of organic liquid scintillation detectors by the normalized average of digitized pulse shapes. Nuclear Instruments and Methods in Physics Research, Section A: Accelerators, Spectrometers, Detectors and Associated Equipment, 2007. 578(1): p. 261-266. 
39. Liu, G., et al., A digital method for the discrimination of neutrons and $\gamma$ rays with organic scintillation detectors using frequency gradient analysis. IEEE Transactions on Nuclear Science, 2010. 57(3 PART 3): p. 1682-1691.

40. Marrone, S., et al., Pulse shape analysis of liquid scintillators for neutron studies. Nuclear Instruments and Methods in Physics Research Section A: Accelerators, Spectrometers, Detectors and Associated Equipment, 2002. 490(1-2): p. 299-307.

41. Kangas, L.J., et al., The use of artificial neural networks in PVT-based radiation portal monitors. Nuclear Instruments and Methods in Physics Research Section A: Accelerators, Spectrometers, Detectors and Associated Equipment, 2008. 587(2): p. 398-412.

42. Nakhostin, M., A new digital method for high precision neutron-gamma discrimination with liquid scintillation detectors. Journal of Instrumentation, 2013. 8(05): p. P05023-P05023.

43. Luo, X.L., et al., Neutron/gamma discrimination employing the power spectrum analysis of the signal from the liquid scintillator BC501A. Nuclear Instruments and Methods in Physics Research Section A: Accelerators, Spectrometers, Detectors and Associated Equipment, 2013. 717: p. 44-50.

44. NEA, O. Java-based nuclear data information system. 16/01/2016 [cited 2016 10/02/2016]; Available from: http://www.oecd-nea.org/janis/.

45. Martin, R.C., J.B. Knauer, and P.A. Balo, Production, distribution and applications of californium-252 neutron sources. Applied Radiation and Isotopes, 2000. 53(4): p. 785-792.

46. Billnert, R., et al., New prompt spectral $\gamma$-ray data from the reaction $252 C f(s f)$ and its implication on present evaluated nuclear data files. Physical Review C - Nuclear Physics, 2013. 87(2).

47. MATLAB and Statistics Toolbox Release 2017b. The MathWorks, Inc.: Natick, Massachusetts, United States. 